\title{
Clinical utility of orally disintegrating olanzapine in Chinese patients with schizophrenia: a review of effectiveness, patient preference, adherence, and other properties
}

\author{
This article was published in the following Dove Press journal: \\ Neuropsychiatric Disease and Treatment \\ 19 February 2014 \\ Number of times this article has been viewed
}

\author{
Jingping Zhao' \\ Jianjun $\mathrm{Ou}^{\prime}$ \\ Haibo Xue ${ }^{2}$ \\ Li Liu ${ }^{2}$ \\ William Montgomery ${ }^{3}$ \\ Tamas Treuer ${ }^{4}$ \\ 'Mental Health Institute of The \\ Second Xiangya Hospital, Hunan \\ Province Technology Institute \\ of Psychiatry, Key Laboratory of \\ Psychiatry and Mental Health of \\ Hunan Province, Central South \\ University, Changsha, Hunan, \\ ${ }^{2}$ Lilly Suzhou Pharmaceutical Co, Ltd, \\ Jiangsu, People's Republic of China; \\ ${ }^{3}$ Global Health Outcomes, Eli Lilly \\ and Company, Sydney, Australia; \\ ${ }^{4}$ Emerging Markets Business Unit \\ (Neuroscience), Eli Lilly and Company, \\ Budapest, Hungary
}

\begin{abstract}
The primary objective of this systematic review was to examine the evidence for the efficacy, effectiveness, and safety of orally disintegrating olanzapine in Chinese populations. A systematic literature search was conducted using databases covering international and Chinese journals, ClinicalTrials.gov, and internal and external trial registries at Eli Lilly and Company using search terms related to target countries (People's Republic of China, Hong Kong, and Taiwan) and orally disintegrating olanzapine treatment. A publication and one clinical study report were retrieved. The clinical study showed orally disintegrating olanzapine and the standard oral tablet to have similar efficacy and tolerability profiles. A bioequivalence study has shown that orally disintegrating olanzapine and the standard oral tablet have similar pharmacokinetic profiles. Orally disintegrating olanzapine and the standard oral tablet have similar efficacy and tolerability profiles.
\end{abstract}

Keywords: orally disintegrating, olanzapine, Chinese, schizophrenia, patients

\section{Introduction}

\section{Epidemiology of schizophrenia and antipsychotic} use in the People's Republic of China

Schizophrenia is a severe mental disorder characterized by a range of cognitive, behavioral, and emotional dysfunctions. It affects around $0.3 \%-0.7 \%$ of people at some point in their lives, ${ }^{1}$ or 24 million people worldwide as of $2011 .{ }^{2}$ Chinese studies show that the lifetime prevalence of schizophrenia is around $0.3 \%-0.97 \%$. Phillips et al studied the prevalence of schizophrenia in four provinces in the People's Republic of China, and reported a lifetime prevalence of $0.78 \% .^{3}$

The primary treatment modality for schizophrenia is antipsychotic medication, in combination with psychological and social supportive care. Currently, most of the atypical antipsychotics, ie, clozapine, olanzapine, risperidone, quetiapine, aripiprazole, ziprasidone, and amisulpride, are available in the People's Republic of China. There are a number of formulations for patients with different needs and preferences, eg, standard tablets, orally disintegrating tablets (ODTs), oral solution, and short-acting and long-acting injectable formulations.

Although liquid and injectable (rapid-acting and long-acting depot) antipsychotics are alternatives to standard tablets in various clinical situations, for example, in the acute setting or where patients have adherence issues, these formulations have limitations.
Correspondence: Jingping Zhao Mental Health Institute of The Second Xiangya Hospital, Hunan Province Technology Institute of Psychiatry, Key Laboratory of Psychiatry and Mental Health of Hunan Province, Central South University, I 39 Middle Renmin Road,

Changsha, Hunan 4100II, People's

Republic of China

Tel +86 73। 85554052

Fax +86 73। 85554052

Email zhaojingpingcsu@।63.com 
Patients may spit out liquid medication, or injectable medication may be unacceptable or contraindicated. Standard tablet forms also have a number of limitations inherent to all standard tablets in the treatment of chronic mental illness. These include administration in situations where the patient is acutely agitated or use in patients with concomitant medical conditions where there is difficulty swallowing. Most importantly, treatment nonadherence (particularly surreptitious/deliberate avoidance) is a major problem in patients with mental illnesses such as schizophrenia. Up to $80 \%$ of patients are at least partially nonadherent to antipsychotic treatment at some point during their illness, which is, in turn, a leading contributor to treatment failure, relapse, and hospitalization. ${ }^{4-6}$

ODTs provide the benefits of a liquid medication in a solid dosage form. Currently in the People's Republic of China, three antipsychotics are available as ODTs, ie, olanzapine (Zyprexa ${ }^{\circledR}$ Zydis $^{\circledR}$, where Zydis refers to the patented freeze-dried orodispersible technology of Catalent Pharma Solutions, Somerset, NJ, USA), risperidone, and aripiprazole (China Food and Drug Administration, http:/www.sda.gov. cn/WS01/CL0001/).

\section{Characteristics of orally disintegrating olanzapine}

An ODT (or orodispersible tablet) differs from a traditional tablet in that it is designed to be dissolved on the tongue/in the mouth rather than be swallowed whole.

The processes used to manufacture ODTs include loose compression tableting, a method that is not very different from that used to manufacture traditional tablets, and lyophilization processes. In loose compression, ODTs are compressed at much lower forces $(4-20 \mathrm{kN})$ than traditional tablets. Lyophilized ODT formulations may use proprietary technologies but can produce a tablet that has a faster disintegration rate; for example, orally disintegrating olanzapine (Zydis) produced by the freeze-drying method consists of olanzapine as the active ingredient entrapped within a matrix of a fast-dissolving carrier material.

Orally disintegrating olanzapine (ODO) tablets are yellow, round, and debossed with the tablet strength. Each ODO tablet contains the olanzapine equivalent of $5 \mathrm{mg}(16 \mu \mathrm{mol})$ or $10 \mathrm{mg}(32 \mu \mathrm{mol})$. US prescribing information is available from http://pi.lilly.com/us/zyprexa-pi.pdf.

ODO tablets dissolve in human saliva in seconds. For example, in a pilot study of eleven patients, where the first measured time points were 15 seconds for initial disintegration and 60 seconds for complete disintegration,
Chue et al found that the mean time to initial disintegration was 15.78 seconds and mean time to complete disintegration was 0.97 minutes. ${ }^{7}$ It has been suggested that ODO may have a faster onset of action and be more appropriate for the treatment of acute agitation in patients with schizophrenia or bipolar disorder.

This paper provides an overview of the characteristics of ODO and reviews the available data on its clinical utility (efficacy, effectiveness, and safety) within the context of the treatment of Chinese patients with schizophrenia or bipolar disorder.

\section{Materials and methods Search strategy and terms}

A prospective systematic literature search strategy to retrieve publications was developed. For the international literature, the electronic databases MEDLINE via PubMed, EMBASE, Cochrane Library, ClinicalTrials.gov, and internal and external trial registries at Eli Lilly and Company were used. For Chinese language publications, the China National Knowledge Infrastructure (http://www.cnki.net), Wan Fang (http://www. wanfangdata.com.cn), and the VIP Information/Chinese Scientific Journals (http://www.cqvip.com) databases were used. The search period was from January 1, 2000 to August 25, 2013. For the Chinese language databases, the search period was from January 1, 2000 to May 29, 2013.

Search terms were chosen relevant to the categories of country, disease state, and treatment using standardized terms where applicable. For the category of country, the search terms were "People's Republic of China", "Chinese", "Taiwan", "Taiwanese", and "Hong Kong". For the category of disease state, the search term was "schizophrenia" or "bipolar disorder". For the category of treatment, the search terms were "orally disintegrating olanzapine", "orally dissolving olanzapine", "orodispersible olanzapine", "Zyprexa Zydis", and "ODT olanzapine”. For all databases, search terms within each category were combined using the Boolean operator OR. Categories were then combined using the Boolean operator AND.

\section{Inclusion and exclusion criteria}

Studies were included if they met the following criteria: Chinese patients with schizophrenia disorder wholly residing in mainland China, Taiwan, or Hong Kong; pharmacokinetic studies of ODO; on-label use of ODO in terms of dose and indication; published evidence from randomized controlled trials, systematic reviews, or observational studies (prospective and retrospective; crossover and noncrossover) 
including cross-sectional studies and longitudinal (eg, cohort) studies. Included studies also had to report numerical data on at least one recognized outcome measure related to efficacy, effectiveness, or safety/tolerability.

Currently unpublished studies sponsored by Eli Lilly were considered for inclusion in this review if they were of reasonable sample size and of sufficient rigor in design to allow meaningful interpretation.

Studies were specifically excluded if they were duplicate publications or contained previously reported data, had no numerically reportable data on at least one relevant outcome measure, did not include olanzapine treatment, or were published evidence from case studies, case series, narrative reviews, editorials, or letters to the editor.

\section{Data extraction}

All authors contributed to the literature search strategy, inclusion/exclusion criteria, and reporting of outcomes. For non-Chinese language publications, one person (not an author) conducted the literature search and screened the title and abstract of publications identified by the search criteria. For Chinese language publications, two Chinese-speaking authors conducted the literature search, and screened the title and abstract of publications identified by the search against the inclusion/exclusion criteria. Chinese language publications were translated into English by one of the Chinese authors and each translation was verified by another author.

\section{Results}

\section{Clinical studies}

Although a number of studies have examined the efficacy and safety, patient adherence, and usability of ODO, studies in Chinese are rare (Figure 1). One study was done in Chinese patients with schizophrenia in Taiwan. The purpose of this study was to compare the efficacy and safety of intramuscular olanzapine, intramuscular haloperidol, olanzapine ODT, and oral risperidone solution for the treatment of agitated patients with psychosis during the first 24 hours of treatment in an acute-care psychiatric ward. Forty-two inpatients from an acute-care psychiatric ward of a medical center in central Taiwan were enrolled. They were randomly assigned to one of the four treatment groups (10 mg intramuscular olanzapine, $10 \mathrm{mg}$ olanzapine ODT, $3 \mathrm{mg}$ oral risperidone solution, or $7.5 \mathrm{mg}$ intramuscular haloperidol). Agitation was measured using the excited component of the Positive and Negative Syndrome Scale (PANSS-EC), the Agitation-Calmness Evaluation Scale, and the Clinical Global Impression of Severity Scale during the first 24 hours. The results showed that there were significant differences in the PANSS-EC total scores for the four intervention groups at 15, 30, 45, 60, 75, and 90 minutes after initiation of treatment. More significant differences were found early on in treatment. In post hoc analyses, patients who received intramuscular olanzapine or olanzapine ODT showed significantly greater improvement in PANSS-EC scores than did patients who received intramuscular haloperidol at points $15,30,45,60,75$, and 90 minutes after injection. These findings suggest that intramuscular olanzapine, olanzapine ODT, and oral risperidone solution were as effective as intramuscular haloperidol for the treatment of patients with acute agitation. Intramuscular olanzapine and olanzapine ODT were more effective than intramuscular haloperidol in the early phase of the intervention; however, there were no significant differences in effectiveness between intramuscular olanzapine, olanzapine ODT, and oral risperidone solution. The most commonly reported and observed adverse effects related to medications were found in all four groups. Drowsiness was most common. Intramuscular olanzapine and ODT olanzapine produced more drowsiness than oral risperidone and intramuscular haloperidol, but the difference was not significant. ${ }^{8}$

A bioequivalence study of two different $5 \mathrm{mg}$ olanzapine tablet formulations in healthy Chinese male subjects was done between May 2007 and June 2007 (Huilin Z. Eli Lilly and Company. A bioequivalence study of two $5 \mathrm{mg}$ olanzapine tablet formulations in healthy Chinese male subjects. Unpublished data, 2008). The primary objective of this study was to establish the bioequivalence of the Zydis olanzapine $5 \mathrm{mg}$ tablet (ODO, test formulation) compared with that of the marketed standard oral $5 \mathrm{mg}$ olanzapine tablet ( reference formulation) in healthy male Chinese subjects. The secondary objective was to collect safety data on the Zydis $5 \mathrm{mg}$ olanzapine tablet formulation in healthy adult male Chinese subjects. The mean plasma concentration versus time curves

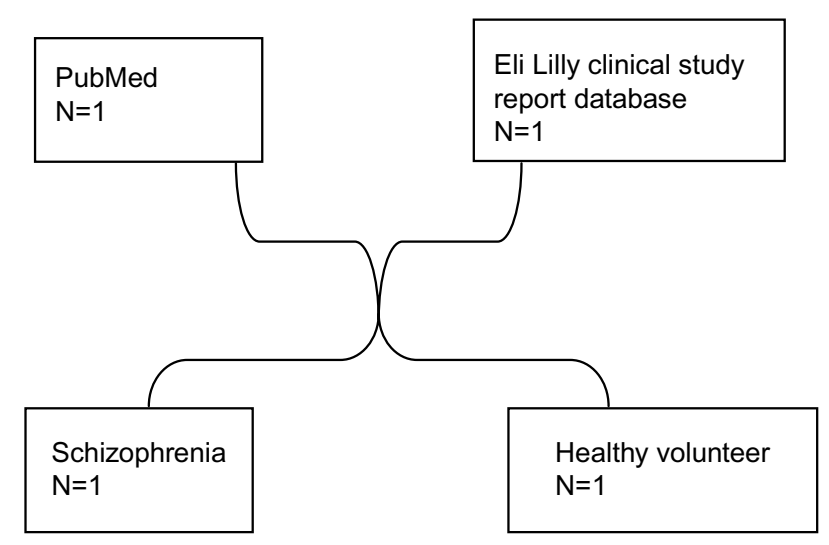

Figure I Publication flow diagram. 
for olanzapine (standard oral tablet, reference formulation) and olanzapine Zydis (ODO, test formulation) treatments were comparable; the ratio of the least squares mean of the area under the curve $(0-\infty)$ and time to maximum concentration were 0.94 and 0.96 , respectively. For both parameters, the $90 \%$ confidence interval was completely contained within the prescribed bioequivalence limits of 0.8 and 1.25; there were no deaths or serious study drug-related adverse events reported in this study. There were no differences in adverse events reported for either olanzapine (standard oral tablet, reference formulation) or olanzapine Zydis (ODO test formulation). The most common adverse event reported was somnolence (Huilin Z. Eli Lilly and Company. A bioequivalence study of two $5 \mathrm{mg}$ olanzapine tablet formulations in healthy Chinese male subjects. Unpublished data, 2008).

\section{Safety}

Because both the ODO and standard oral tablet formulation contain olanzapine as the active ingredient, they were expected to have similar safety and tolerability profiles. Indeed, the tolerability profile seen in clinical studies with ODO has been found to be similar to the more extensively studied standard oral tablet formulation. A number of studies of ODO have included measurement of safety and tolerability. In the bioequivalence study of ODO and the standard oral tablet in healthy male Chinese subjects, a total of 38 adverse events were reported for 19 of 24 subjects who received the study drug. Thirty-three adverse events (in 18 subjects) were judged by the investigator to be possibly/ probably related to the study drug (Huilin Z. Eli Lilly and Company. A bioequivalence study of two $5 \mathrm{mg}$ olanzapine tablet formulations in healthy Chinese male subjects. Unpublished data, 2008). All events related to the study treatment were of mild severity except for one episode of "orthostatic hypotension" (subject No. 120, after olanzapine Zydis) that was rated as moderately severe. The most common adverse event reported was somnolence. No deaths or other serious adverse events occurred during this study (Huilin Z. Eli Lilly and Company. A bioequivalence study of two $5 \mathrm{mg}$ olanzapine tablet formulations in healthy Chinese male subjects. Unpublished data, 2008).

\section{Extrapyramidal symptoms}

There have been no studies investigating extrapyramidal symptoms associated with ODO in Chinese patients. Other studies have shown that ODO has a tendency to produce less extrapyramidal symptoms, but there were no significant differences when ODO was compared directly with the standard oral tablet. ${ }^{7,9}$

\section{Weight gain and metabolic profile}

ODO may be absorbed via the buccal mucosa or before reaching the pylorus (particularly when administered sublingually), and it has been hypothesized that this may lead to a different weight change profile compared with the standard oral tablet. However, there has been no study conducted to investigate the weight gain and metabolic changes associated with ODO in Chinese patients. Such studies are needed in the future. The currently available evidence regarding a difference in the potential for weight gain with ODO versus the standard oral tablet is inconsistent and warrants further study. However, what does seem apparent is that if a difference exists between these two formulations in this regard, it is not likely to be a substantial one and may manifest only in certain patient populations.

\section{Nursing burden}

Schizophrenia and bipolar disorder impose a significant work burden on nursing staff in inpatient treatment settings where treatment often needs to be administered under difficult circumstances and where patients are closely monitored on an ongoing basis. Eli Lilly and Company have developed the Nursing Assessment of Medication Acceptance (NAMA) scale to systematically investigate acceptance and attitude towards medication, including assessing the burden of care on nursing staff. ${ }^{9}$ The NAMA questionnaire includes four items, ie, attitude (patient has a positive attitude towards medication), compliance (patient complies with medication intake), ingestion (patient ingests medication), and nursing (no extensive nursing effort is needed to administer medication), from which a total score may be calculated. There has been no study of the nursing burden in Chinese patients. Kinon et al used the NAMA questionnaire to assess the nursing burden of treatment with ODO in acutely ill noncompliant patients with schizophrenia and schizoaffective disorder. Significant improvements in nursing burden were seen as early as day 2 and improvements generally continued through to week $6 .^{9}$

\section{Clinical utility}

ODO is currently available in 12 provinces in the People's Republic of China and is included on the provincial reimbursement drug list in ten provinces. Sixty-three hospitals in the People's Republic of China have ODO available. 
In practice, ODO has been used primarily in patients with first-episode schizophrenia, patients already stabilized on treatment, patients with diagnoses of schizophrenia, schizoaffective, schizophreniform disorder, or bipolar disorder, and those who are known to be nonadherent with treatment. The ODO formulation may have some advantages, not only where medical conditions present swallowing difficulties with standard tablets (eg, dysphagia, stroke, gastroesophageal reflux disease) and in situations where access to liquids or discretion is needed, but also where a rapid effect (eg, agitated patients in emergency settings) and/or where medication adherence is considered to be a potential concern or medication administration needs to be verified. The choice of ODO over the standard oral tablet would seem particularly prudent when there is a change in treatment setting which may impact adherence, such as moving from a more controlled environment to a less controlled one, eg, discharge from an inpatient facility or change in caregiver or accommodation.

\section{Conclusion}

This paper reviews the clinical utility of ODO in Chinese patients. The existing data suggest that although ODO has an efficacy and tolerability profile similar to that of the standard oral tablet, long-term patient outcomes may be improved with ODO in some patients due to better adherence, leading to a lower risk of relapse/hospitalization. ODO may also have benefits over the standard oral tablet in terms of nursing burden and patient preference, and this should be taken into consideration when making treatment decisions. However, clinical studies in Chinese patients are limited. Further studies are needed to answer clinical questions on the effectiveness, adherence, and preference of ODO.

\section{Disclosure}

HX, LL, WM, and TT are employees of Eli Lilly and Company, manufacturer of olanzapine in standard tablet form (Zyprexa) and orally disintegrating form (Zyprexa Zydis11/ Velotab $\left.^{\mathrm{TM}}\right)$. The remaining authors have no conflicts of interest to declare.

\section{References}

1. Van Os J, Kapur S. Lancet. 2009;374:635-645.

2. World Health Organization. Schizophrenia. Available from: http://www. who.int/mental_health/management/schizophrenia/en/.Accessed February $27,2011$.

3. Phillips M, Zhang JX, Shi QC, et al. Prevalence, treatment, and associated disability of mental disorders in four provinces in China during 2001-2005: an epidemiological survey. Lancet. 2009;373:2041-2053.

4. Leucht S, Barnes TR, Kissling W, Engel RR, Correll C, Kane JM. Relapse prevention in schizophrenia with new generation antipsychotics: a systematic review and exploratory meta analysis of randomized, controlled trials. Am J Psychiatry. 2003;160:1209-1222.

5. Corrigan PW, Liberman RP, Engel JD. From noncompliance to collaboration in the treatment of schizophrenia. Hosp Community Psychiatry. 1990;41:1203-1211.

6. Lacro JP, Dunn LB, Dolder CR, Leckband SG, Jeste DV. Prevalence of and risk factors for medication nonadherence in patients with schizophrenia: a comprehensive review of recent literature. J Clin Psychiatry. 2002;63:892-909.

7. Chue P, Jones B, Taylor CC, Dickson R. Dissolution profile, tolerability, and acceptability of the orally disintegrating olanzapine tablet in patients with schizophrenia. Can J Psychiatry. 2002;47:771-774.

8. Hsu WY, Huang SS, Lee BS, Chiu NY. Comparison of intramuscular olanzapine, orally disintegrating olanzapine tablets, oral risperidone solution, and intramuscular haloperidol in the management of acute agitation in an acute care psychiatric ward in Taiwan. J Clin Psychopharmacol. 2010;30:230-234.

9. Kinon BJ, Hill AL, Liu H, Kollack-Walker S. Olanzapine orally disintegrating tablets in the treatment of acutely ill non-compliant patients with schizophrenia. Int J Neuropsychopharmacol. 2003;6: 97-102.
Neuropsychiatric Disease and Treatment

\section{Publish your work in this journal}

Neuropsychiatric Disease and Treatment is an international, peerreviewed journal of clinical therapeutics and pharmacology focusing on concise rapid reporting of clinical or pre-clinical studies on a range of neuropsychiatric and neurological disorders. This journal is indexed on PubMed Central, the 'PsycINFO' database and CAS

\section{Dovepress}

The manuscript management system is completely online and includes a very quick and fair peer-review system, which is all easy to use. Visit http://www.dovepress.com/testimonials.php to read real quotes from published authors. 